\title{
A New Stabilized Nodal Integration Approach
}

M. Puso, E. Zywicz, J. S. Chen

February 13, 2006

Third International Workshop Meshfree Methods for Partial Differential Equations Bonn Germany, Germany September 12, 2005 through September 15, 2005 
This document was prepared as an account of work sponsored by an agency of the United States Government. Neither the United States Government nor the University of California nor any of their employees, makes any warranty, express or implied, or assumes any legal liability or responsibility for the accuracy, completeness, or usefulness of any information, apparatus, product, or process disclosed, or represents that its use would not infringe privately owned rights. Reference herein to any specific commercial product, process, or service by trade name, trademark, manufacturer, or otherwise, does not necessarily constitute or imply its endorsement, recommendation, or favoring by the United States Government or the University of California. The views and opinions of authors expressed herein do not necessarily state or reflect those of the United States Government or the University of California, and shall not be used for advertising or product endorsement purposes. 


\title{
A New Stabilized Nodal Integration Approach
}

\author{
Michael Anthony Puso ${ }^{1}$, Edward Zywicz ${ }^{2}$, and J.S. Chen ${ }^{3}$ \\ 1 Lawrence Livermore National Laboratory, Livermore CA 94550 puso@llnl.gov \\ 2 Lawrence Livermore National Laboratory, Livermore CA 94550 \\ zywicz@llnl.gov \\ 3 University of California at Los Angeles, Los Angeles CA 90095 \\ jschen@seas.ucla.edu
}

Summary. A new stabilized nodal integration scheme is proposed and implemented. In this work, focus is on the natural neighbor meshless interpolation schemes. The approach is a modification of the stabilized conforming nodal integration (SCNI) scheme and is shown to perform well in several benchmark problems.

Key words: Nodal integration, natural neighbor, particle method

\section{Introduction}

Nodal integration of the Galerkin weak form of the equations of motion is often desirable due to its efficiency and applicability in large deformation problems. For example, in the large deformation case, the stress and material history will move with the nodes and so no re-mapping of the quantities is required. Numerous formulations and types of meshless shape functions are available, here, we will focus on Galerkin formulations where natural neighbor $(\mathrm{NN})$ interpolation is used; often referred to as the natural element method (NEM) [7]. Different nodal integration approaches have been developed such as stabilized conforming nodal integration (SCNI) $[3,10,5]$ and a least squares stabilization approach [1]. The latter approach was applied using an EFG formulation and suggested the use of quadratic basis functions due to the second derivatives in the least squares term. Furthermore, the least squares formulation in [1] is not directly applicable to natural neighbor schemes since they are not smooth at the nodes. Recently we have found that the SCNI method applied to NN shape functions produces spurious low energy (not zero energy) modes in some problems and these modes did not appear to vanish with refinement. In this work, a modification to the SCNI method is made that appears to provide stable results as demonstrated in eigenvalue and large deformation examples provided here.

Natural neighbor (NN) shape functions have some distinct advantages over many meshless schemes in that they interpolate the data making it simpler 
to implement essential boundary conditions and the nodal adjacency only includes near neighbors. The NN/NEM approach has been implemented in the context of a meshless method by exploiting the concept of alpha shapes $[4,6]$ to determine/treat the free surface of cloud of points. In this work, the integration method in $[10,5]$ is modified by applying an additional stabilization term. In what follows: Section 2 introduces the global weak form, the NN interpolation scheme and the SCNI approach with added stabilization, Section 3 presents results demonstrating the necessity of the added stabilization and effectiveness of the proposed approach.

\section{Formulation}

The formulation will be introduced in the context of linear elasticity and the straightforward extensions to the nonlinear regime will be given at the end of the section.

\subsection{Galerkin Method}

Considering a body occupying the domain $\Omega$, the governing equations of motion are given

$$
\rho \ddot{\boldsymbol{u}}=\nabla \boldsymbol{\sigma}+\boldsymbol{b}
$$

where $\boldsymbol{u}$ is the displacement field, $\boldsymbol{b}$ is the body force and $\boldsymbol{\sigma}=\boldsymbol{\sigma}(\boldsymbol{\varepsilon})$ is the Cauchy stress in terms of strain

$$
\varepsilon(\boldsymbol{u})=1 / 2\left(\nabla \boldsymbol{u}+\nabla \boldsymbol{u}^{T}\right)
$$

Employing test function $\boldsymbol{v}$, the weak form of (1) can then be written

$$
\int_{\Omega} \rho \boldsymbol{v} \cdot \ddot{\boldsymbol{u}} d \Omega+\int_{\Omega} \boldsymbol{\varepsilon}(\boldsymbol{v}): \boldsymbol{\sigma}(\varepsilon(\boldsymbol{v})) d \Omega=\int_{\Omega} \boldsymbol{v} \cdot \boldsymbol{b} d \Omega+\int_{\Gamma_{t}} \boldsymbol{v} \cdot \boldsymbol{t} d \Gamma
$$

where applied tractions $\boldsymbol{t}$ are specified on the boundary $\boldsymbol{\Gamma}_{t}$.

\subsection{Discrete Form}

Following standard procedure, the discrete displacement field is defined

$$
\boldsymbol{u}_{h}=\sum_{I=1}^{N} \phi_{I}(\boldsymbol{x}) \boldsymbol{u}_{I} \quad \boldsymbol{x} \in \Omega_{h}
$$

in terms of shape functions $\phi_{I}$ and nodal displacements $\boldsymbol{u}_{I}$ over all nodes $I=1, N$ on the discretized domain $\Omega_{h}$. Unlike finite elements, the definition of the domain $\Omega_{h}$ in meshless methods is not straightforward and is defined 
in what follows. Substituting (4) into the weak form (3) and applying nodal integration, the following discrete form results

$$
\sum_{I=1}^{N} m_{I} \boldsymbol{v}_{I} \cdot \ddot{\boldsymbol{u}}_{I}+V_{I} \boldsymbol{\varepsilon}_{I}\left(\boldsymbol{v}_{h}\right): \boldsymbol{\sigma}\left(\boldsymbol{\varepsilon}_{I}\left(\boldsymbol{u}_{h}\right)\right)-\boldsymbol{v}_{I} \cdot \boldsymbol{f}_{I}=0
$$

where $m_{I}$ is the nodal mass, $f_{I}$ is a collocated form of the applied loads, $\varepsilon_{I}$ is the nodal strain and $V_{I}$ is the nodal volume. Since the NN shape functions interpolate the data, the nodal mass is simply $m_{I}=\rho V_{I}$. The body force contribution to $\boldsymbol{f}_{I}$ is computed similarly. Here, the traction force contribution to $f_{I}$ is computed using the surface mesh but other techniques are outlined in [1]. The $\boldsymbol{\varepsilon}_{I}$ could be the symmetric gradient of the displacement at $\boldsymbol{x}_{I}$ or computed using SCNI as shown in what follows. In this work, $V_{I}$ represents the volume of the Voronoi cell $\Omega_{I}$ about node $I$ (Fig. 1(a)). The Voronoi diagram of a set of sites $P:=\left\{\boldsymbol{x}_{1}, \boldsymbol{x}_{2}, \ldots \boldsymbol{x}_{N}\right\}$ is a partition such that all points $\boldsymbol{x}$ within a Voronoi cell $\Omega_{I}$ are closer to the generating site $I$ than to any other site in $P$. Considering three dimensional space, this can be mathematically stated

$$
\Omega_{I}=\left\{\boldsymbol{x} \in \mathbb{R}^{3}: d\left(\boldsymbol{x}, \boldsymbol{x}_{I}\right)<d\left(\boldsymbol{x}, \boldsymbol{x}_{J}\right) \forall J \neq I\right\}
$$

To handle arbitrary non-convex geometries, the alpha - shapes approach is used to compute a surface mesh from the cloud of points. The Voronoi diagram is then "clipped" by the surface in an approach similar to that in [4] and [6] thus producing the discretized domain $\Omega_{h}$. Figure 1(b) illustrates this process for two dimensions and our implementation performs the equivalent in three dimensions. Of course, this approach may be too computationally intensive for some applications and one may resort to simpler approaches to compute nodal volumes (c.f. [1]).

\subsection{Natural Neighbor Interpolation}

The Voronoi cell for point $I$ is formed by polygonal (usually triangular) cell walls and the points with common cell walls are the set of natural (or nearest) neighbors $\mathcal{N}\left(\boldsymbol{x}_{I}\right)$ for point $I$. The Laplace (or non-Sibson) form of NN interpolation [8] computes interpolation functions at a point $\boldsymbol{x}$ in the following way. Consider the Voronoi diagram shown in black in Fig. 2, the interpolation function $\phi$ at a point $\boldsymbol{x}$ is computed by doing a point insertion where $\boldsymbol{x}$ is treated as an additional vertex in the set $P$ and a new Voronoi cell is formed (shown in blue in Fig. 2(a)). For the two dimensional case, the heights $h$ and cell wall lengths $s$ are used to compute the shape functions evaluated at point $\boldsymbol{x}$

$$
\phi_{J}(\boldsymbol{x})=\frac{s_{J} / h_{J}}{\sum_{K \in \mathcal{N}(\boldsymbol{x})} s_{K} / h_{K}} \quad \forall J \in \mathcal{N}(\boldsymbol{x}) .
$$

In three dimensions, the quantity $s$ is a cell wall area. The NN interpolation functions are both linear exact and interpolative. 


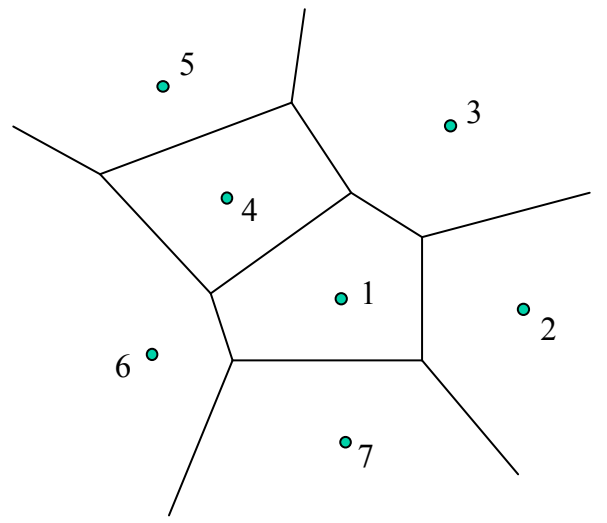

(a)

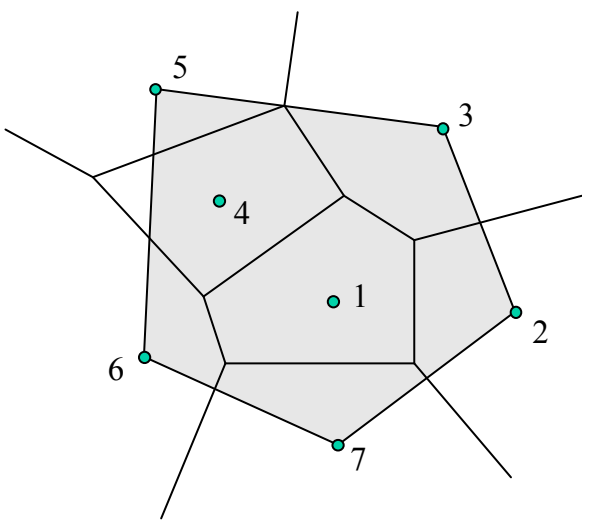

(b)

Figure 1. (a) Voronoi diagram about cloud of points. (b) Surface mesh and clipped Voronoi cells.

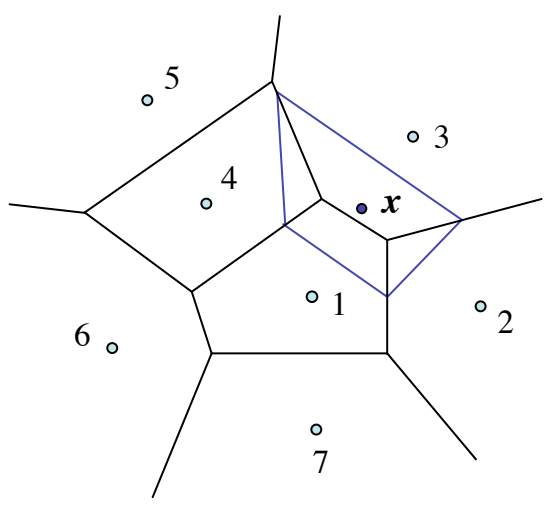

(a)

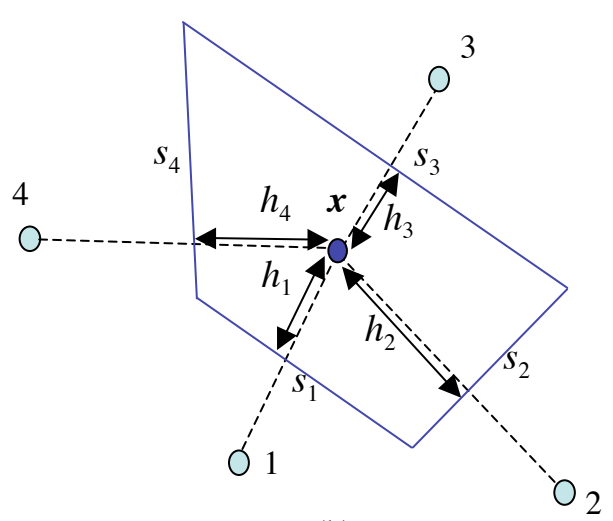

(b)

Figure 2. (a) Point insertion at $x$. (b) Geometry of Voronoi cell about $\boldsymbol{x}$

\subsection{Stabilized Conforming Nodal Integration (SCNI)}

If smooth shape functions are used, one could merely take derivatives at the nodes to compute the nodal strain $\varepsilon_{I}$ (although patch test satisfaction may be violated). Since NN shape functions are not smooth, a different approach is required. Here, the nodal strain is computed using the SCNI approach which is well described in $[3,10,5]$. In short, $\boldsymbol{\varepsilon}_{I}$ is computed from the volume average of the strain $\varepsilon$ over the Voronoi cell domain $\Omega_{I}$ and using divergence theorem results in the surface integral

$$
\boldsymbol{\varepsilon}_{I}\left(\boldsymbol{u}_{h}\right)=\frac{1}{V_{I}} \int_{\partial \Omega_{I}}\left(\boldsymbol{u}_{h} \otimes \boldsymbol{n}+\boldsymbol{n} \otimes \boldsymbol{u}_{h}\right) / 2 d \Gamma
$$


where $\boldsymbol{n}$ is the unit normal along the contour surface of cell $I$. Numerical integration is employed in computing (8) such that one integration point at the centroid of each Voronoi facet is used. This approach exactly satisfies patch test and can be used without any additional stabilization in many situations as demonstrated in $[3,10,5]$. On the other hand, it is shown here that problems arise such that spurious low energy modes arise in some applications of SCNI motivating the following approach.

\subsection{Modified SCNI}

As mentioned, the SCNI formulation produces spurious modes as seen in Fig. 4(b). Although the SCNI approach does not have zero energy modes, a straightforward analysis can demonstrate that the method is not $V_{1}$ coercive. Referring to (3) and (5), the discrete bilinear form for linear elasticity is written

$$
a\left(\boldsymbol{v}_{h}, \boldsymbol{u}_{h}\right)=\sum_{I=1}^{N} \varepsilon_{I}\left(\boldsymbol{v}_{h}\right): \boldsymbol{C} \varepsilon_{I}\left(\boldsymbol{u}_{h}\right) V_{I}
$$

where $C$ is the elasticity tensor and the discrete strain energy results when $\boldsymbol{v}_{h} \doteq \boldsymbol{u}_{h}$. Considering a one dimensional BVP with evenly spaced nodes, the appropriate saw tooth displacement field would cause the nodal strain defined by (8) to be zero everywhere except at the boundaries. This would produce a zero energy mode for an infinite domain and a spurious low energy mode for a finite domain. In order to eliminate these modes the Voronoi cells at node $I$ are further decomposed into sub-cells $\Omega_{I}^{c}$ as shown for the two dimensional case in Fig. 3. Here each sub-cell is a triangle/polyhedron formed from the generating node and a Voronoi edge/facet in two/three dimensions respectively. In practice, we can merge adjacent triangle/polyhedron into reduced set of sub-cells. Examples in this work use eight sub-cells by performing this merge process although some nodes may actually have less than eight subcells since they have less than eight Voronoi facets. The same SCNI approach is used to compute the strain over each sub-cell $\Omega_{I}^{c}$

$$
\boldsymbol{\varepsilon}_{I}^{c}\left(\boldsymbol{u}_{h}\right)=\frac{1}{V_{I}^{c}} \int_{\partial \Omega_{I}^{c}}\left(\boldsymbol{u}_{h} \otimes \boldsymbol{n}+\boldsymbol{n} \otimes \boldsymbol{u}_{h}\right) / 2 d \Gamma \quad c=1: N_{I}^{c}
$$

where $N_{I}^{c}$ is the number of sub-cells used at node $I$ and $V_{I}^{c}$ is the volume of the sub-cell. The modified SCNI weak form is now proposed

$$
\begin{aligned}
a\left(\boldsymbol{v}_{h}, \boldsymbol{u}_{h}\right)=\sum_{I=1}^{N}\left[\varepsilon_{I}\left(\boldsymbol{v}_{h}\right): \boldsymbol{C} \boldsymbol{\varepsilon}_{I}\left(\boldsymbol{u}_{h}\right) V_{I}+\right. \\
\left.\sum_{c=1}^{N_{I}^{c}} \alpha_{I}^{c}\left(\boldsymbol{\varepsilon}_{I}\left(\boldsymbol{v}_{h}\right)-\boldsymbol{\varepsilon}_{I}^{c}\left(\boldsymbol{v}_{h}\right)\right): \tilde{\boldsymbol{C}}\left(\boldsymbol{\varepsilon}_{I}\left(\boldsymbol{u}_{h}\right)-\boldsymbol{\varepsilon}_{I}^{c}\left(\boldsymbol{u}_{h}\right)\right) V^{c}\right]
\end{aligned}
$$


where $\alpha_{I}^{c}$ is a penalty parameter and $\tilde{C}$ is the elasticity tensor or some modified version. In the proceeding examples, the sub-cell penalty will be uniform i.e. $\alpha_{I}^{c}=1$ amounting to what is sometimes referred to as "physical stabilization" $[2,9]$. Here, the stiffness $\tilde{\boldsymbol{C}}$ is chosen in the following way:

Isotropic elastic material: Lamé parameters $\mu$ and $\lambda$ :

$$
\tilde{\mu}:=\mu \quad \tilde{\lambda}:=\max (\lambda, 25 \tilde{\mu})
$$

Isotropic plastic material: Lamé parameter $\lambda$ and plastic modulus $E_{T}$ :

$$
\tilde{\mu}:=E_{T} / 2 \quad \tilde{\lambda}:=\max (\lambda, 25 \tilde{\mu})
$$

For non-isotropic materials, the choice for $\tilde{\mu}$ and $\tilde{\lambda}$ would be less obvious but still tractable. Using a maximum in (12) and (13) mitigates volumetric locking. The nodal deformation gradients $\boldsymbol{F}_{I}, \boldsymbol{F}_{I}^{c}$ are defined

$$
\boldsymbol{F}_{I}\left(\boldsymbol{u}_{h}\right)=\frac{1}{V_{I}} \int_{\partial \Omega_{I}} \boldsymbol{u}_{h} \otimes \boldsymbol{n} d \Gamma \quad \boldsymbol{F}_{I}^{c}\left(\boldsymbol{u}_{h}\right)=\frac{1}{V_{I}^{c}} \int_{\partial \Omega_{I}^{c}} \boldsymbol{u}_{h} \otimes \boldsymbol{n} d \Gamma \quad e=1: N_{I}^{c}
$$

and for large deformation kinematics, the internal virtual work (11) is redefined

$$
\begin{aligned}
a\left(\boldsymbol{v}_{h}, \boldsymbol{u}_{h}\right)=\sum_{I=1}^{N} & {\left[\boldsymbol{F}_{I}\left(\boldsymbol{v}_{h}\right): \boldsymbol{P}\left(\boldsymbol{F}_{I}\left(\boldsymbol{u}_{h}\right)\right) V_{I}+\right.} \\
& \left.\sum_{e=1}^{N_{I}^{c}} \alpha_{I}^{c}\left(\boldsymbol{F}_{I}\left(\boldsymbol{v}_{h}\right)-\boldsymbol{F}_{I}^{c}\left(\boldsymbol{v}_{h}\right)\right): \tilde{\boldsymbol{C}}\left(\boldsymbol{F}_{I}\left(\boldsymbol{u}_{h}\right)-\boldsymbol{F}_{I}^{c}\left(\boldsymbol{u}_{h}\right)\right) V^{c}\right]
\end{aligned}
$$

where $\boldsymbol{P}$ is the first Piola Kirchoff stress and $V_{I}$ and $V_{I}^{c}$ are determined in the initial reference configuration. The numerical implementation replaces the internal virtual work (9) with (11) or (15) in the weak form of the equations of motion (3).

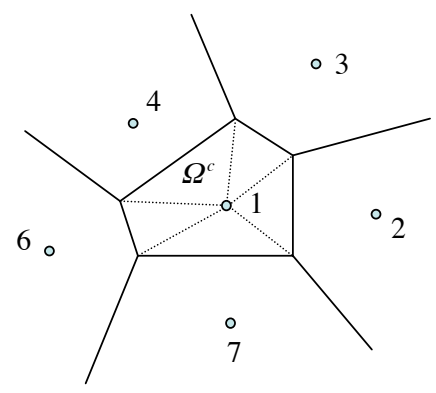

Figure 3. Subcells in the Voronoi cell formed from $\boldsymbol{x}_{I}$. 


\subsection{Implementation Details}

In our implementation, the strain displacement matrices were computed at initialization and then stored to avoid re-computation of the Voronoi diagram at every time step. This also applies to the nonlinear kinematics case where the total Lagrangian approach (15) was employed.

The SCNI and the stabilization approach require the computation of shape functions at the Voronoi cell walls and polygons separating adjacent sub-cells for each node $I$. To expedite this calculation and to reduce connectivity, the smallest set of nodes were employed in evaluating shape functions:

1. Only the set $\mathcal{N}\left(\boldsymbol{x}_{I}\right) \cup \mathcal{N}\left(\boldsymbol{x}_{J}\right)$ was used for Voronoi cell wall shape function evaluations where the cell wall was common to the Voronoi cells of node $\boldsymbol{x}_{I}$ and $\boldsymbol{x}_{J}$.

2. Only the set $\mathcal{N}\left(\boldsymbol{x}_{I}\right)$ was used for the interior sub-cell shape function evaluations.

The above almost always worked and one could probably even use less nodes. If the approach didn't work (i.e. the cell wasn't closed), more nodes were added to the set based on adjacency. Unlike changing the smoothing length of the weight functions with RKPM or EFG to get the appropriate amount of cover, adding nodes to the set to compute shape functions does not affect any previous computations.

The results here used two dimensional triangular finite element interpolation everywhere on the exterior boundary surface mesh to do shape function evaluations along the boundary. We have recently implemented two dimensional Laplace shape functions but have yet to compare the difference.

\section{Results}

The following results demonstrate the necessity and effectiveness of the additional stabilization. Furthermore, the proposed approach satisfies patch tests to machine precision and does not appear to lock in nearly incompressible situations. The constant pressure Q1P0 finite element is used for comparison since it performs reasonably well for the nearly incompressible case.

\section{Eigenanalysis}

This example illustrates the spurious modes using the standard SCNI approach and the improved behavior using additional stabilization. An eigenanalysis of a nearly incompressible $1 \times 1 \times 1$ block $(E=1, \nu=0.499, \rho=1)$ was performed and the first eigenmodes using the Q1P0 brick element, the SCNI NN approach and the proposed approach are plotted in Fig. 4. The frequency versus eigenmode is plotted in Fig. 5 and it is seen that results from the new approach are nearly identical to that of the Q1P0 for the first 100 modes. With SCNI, the frequencies for the first one hundred modes are low due to the presence of spurious modes. 

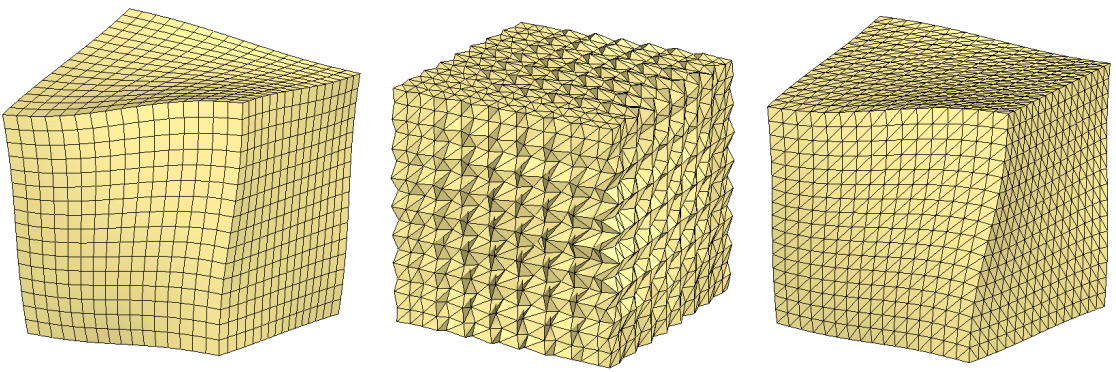

Figure 4. First eigenmode using: (a) brick elements, (b) SCNI (c) modified SCNI.

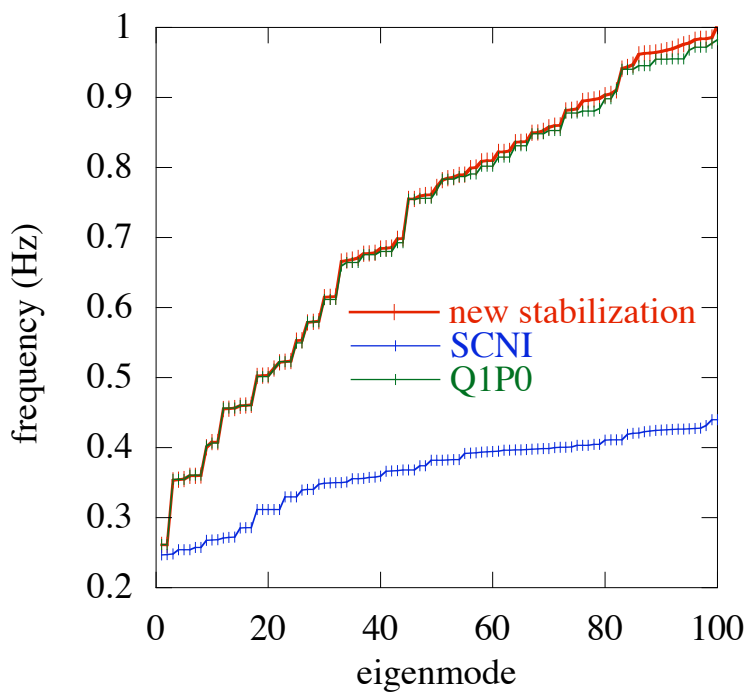

Figure 5. Frequency versus eigenmode for three approaches.

\section{Rubber Billet}

In this example, the performance on a large deformation problem is evaluated. A rubber billet 50.8 in length and diameter (Fig. 6(a)) with Neo Hookean material parameters $(E=1, \nu=0.49)$ was compressed to $70 \%$ strain and only the bottom section was modeled due to symmetry. In the model, a unilateral contact surface was used to vertically constrain the outer surface as it expands (bulges) horizontally. The sequence of deformations from the proposed approach are shown in Fig. 6(a-c) . The SCNI analysis terminated at $41 \%$ strain due to large oscillations (Fig. 6(d)) whereas the stress and deformation match nicely for the proposed approach and the Q1P0 hex element results at this strain threshold (Fig. 6(b,e)). Oscillations do become apparent in the last state shown in Fig. 6(c) compared to the Q1P0 results Fig. 6(e). The total 
force applied to the billet versus strain is shown plotted in Fig. 7 and it seen that the proposed approach matches the Q1P0 quite well.

(a)

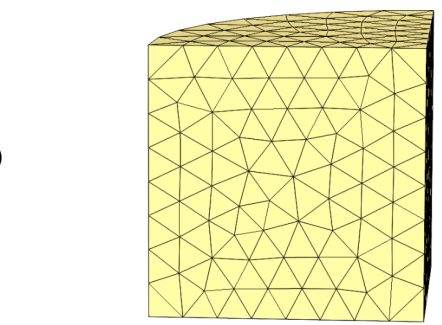

(b)

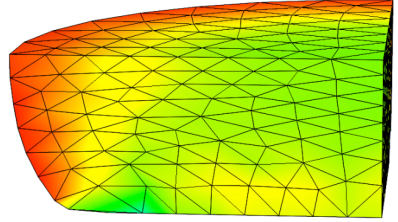

(c)

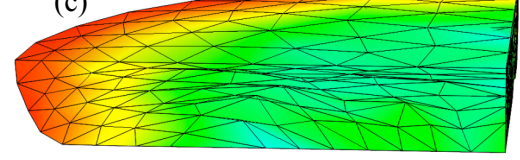

(d)

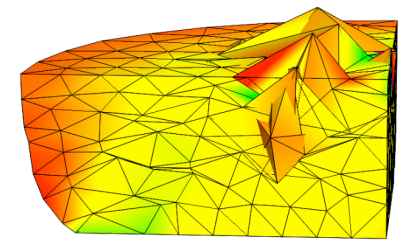

(e)
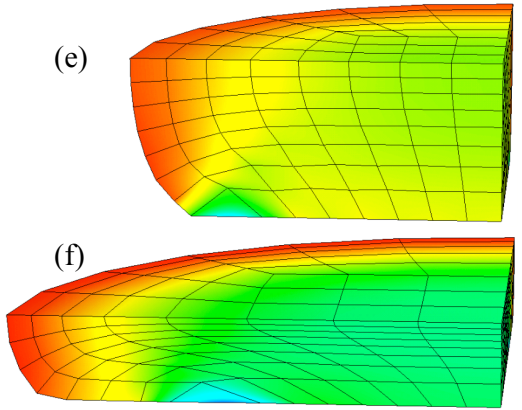

Figure 6. Rubber billet: (a-c) sequence of deformation of stabilized NEM (d) unstabilized NEM (e-f) deformed configurations of Q1P0. The vertical $\sigma_{z}$ stress is indicated in (b-f).

\section{Taylor Bar}

A standard benchmark for plasticity is the Taylor bar impact problem. Here, a copper bar impacts a rigid wall at high velocity. The following elastoplastic material properties were used: $E=117 \mathrm{GPa}, \nu=0.35, \sigma_{y}=0.4 \mathrm{GPa}$, linear hardening modulus $E_{T}=0.1 \mathrm{GPa}$ and density $\rho=8930 \mathrm{~kg} / \mathrm{m}^{3}$. The initial bar length was $32.4 \mathrm{~mm}$, the initial radius was $3.2 \mathrm{~mm}$ and the initial velocity was $227 \mathrm{~m} / \mathrm{s}$. The final deformed states are shown in Fig. 8 where the color indicates displacement magnitude. Again, the new stabilization compares well with the Q1P0 finite element result but the SCNI shows oscillations.

\section{Discussion}

A new stabilized nodal integration approach was developed and applied to the natural neighbor meshless method. The approach performed well in eigenvalue and nonlinear benchmark problems. Here, a total Lagrangian approach was used for large deformation. Extension of the method using an "Eulerian" type 


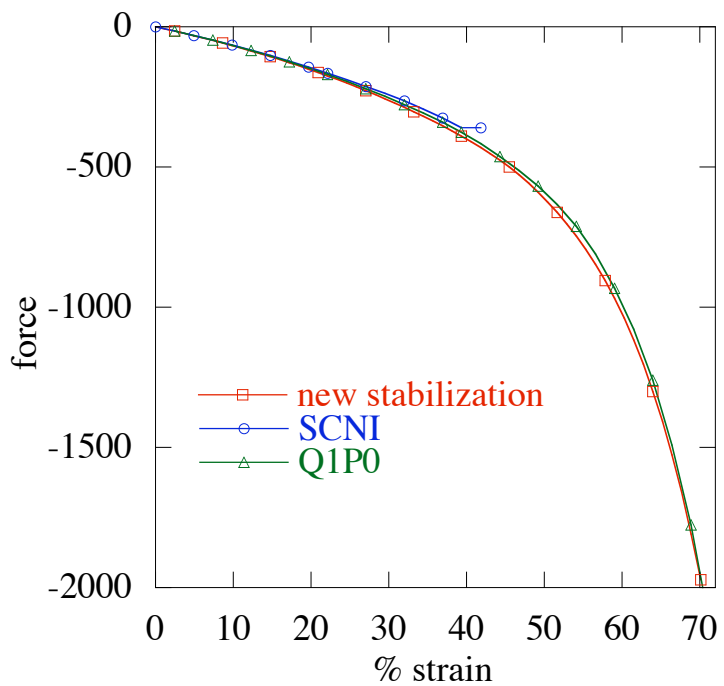

Figure 7. Force versus percent strain for rubber billet

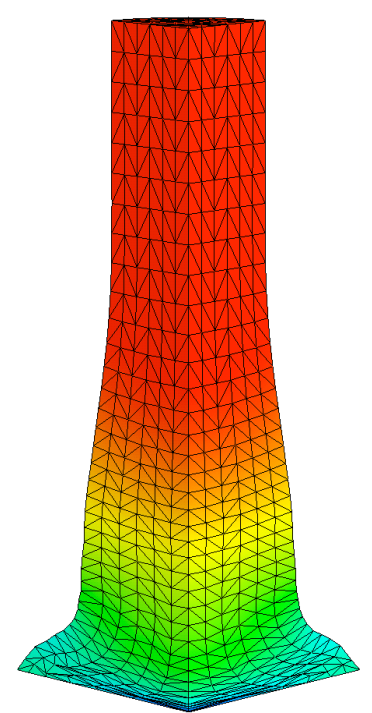

(a)

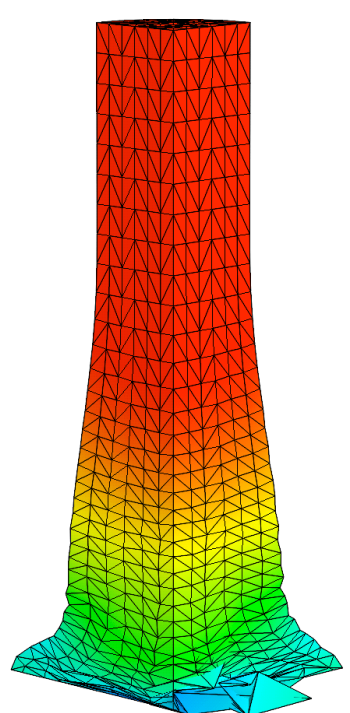

(b)

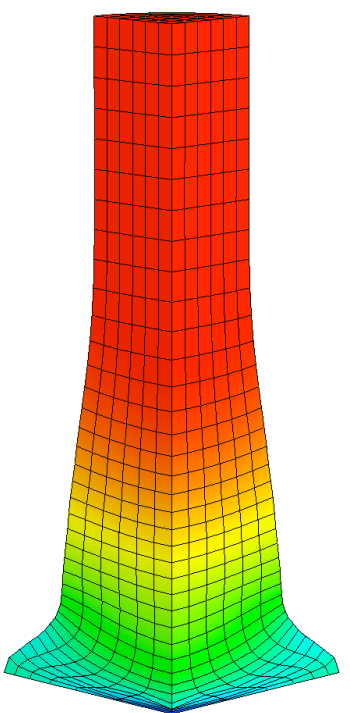

(c)

Figure 8. Final configuration and plot of displacement magnitude from (a) new stabilized (b) SCNI and (c) Q1P0 approaches. 
kernel for extremely large deformations occurring in penetration will be investigated. The method appeared to perform well for the nearly incompressible case but would require modifications to treat the fully incompressible case.

\section{References}

1. S. Beissel and T. Belytschko, Nodal integration of the element-free Galerkin method, Computer Methods in Applied Mechanics and Engineering 139 (1996), 49-74.

2. T. Belytschko and Bindeman L.P., Assumed strain stabilization of the eight node hexahedral, Computer Methods in Applied Mechanics and Engineering 105 (1993), 225-260.

3. J.S. Chen, C.T. Wu, S. Yoon, and Y. You, A stabilized conforming nodal integration for Galerkin mesh-free methods, International Journal for Numerical Methods in Engineering 50 (2001), 435-466.

4. E. Cueto, B. Calvo, and M. Doblare, Modeling three-dimensional piece-wise homogenous domains using the $\alpha$-shape based Natural Element Method, International Journal for Numerical Methods in Engineering 54 (2002), 871-897.

5. D. Gonzalez, E. Cueto, M.A. Martinez, and M. Doblare, Numerical integration in Natural neighbor Galerkin methods, International Journal for Numerical Methods in Engineering 60 (2003), 2077-2104.

6. S. Idelsohn, E. Onate, N. Calvo, and F. Del Pin, The meshless finite element method, International Journal for Numerical Methods in Engineering 58 (2003), 893-912.

7. Sukumar N., Moran B., and Belytschko T., The Natural element method in solid mechanics, International Journal for Numerical Methods in Engineering 43 (1998), 839-887.

8. Sukumar N., Moran B., Yu Semenov Y., and Belikov V.V., Natural neighbor Galerkin methods, International Journal for Numerical Methods in Engineering 50 (1998), 1-27.

9. M.A. Puso, A highly efficient enhanced assumed strain physically stabilized hexahedral element, International Journal for Numerical Methods in Engineering 49 (2003), 1024-1064.

10. J.W. Yoo, B. Moran, and J.S. Chen, Stabilized conforming nodal integration in the Natural Element Method, International Journal for Numerical Methods in Engineering 60 (2004), 861-890.

\section{This work was performed under the auspices of the U.S. Department of Energy by University of California, Lawrence Livermore National Laboratory under Contract W-7405-Eng-48.}

